\title{
Chapter Neuroimaging the effects of light on non-visual brain functions
}

\author{
Gilles Vandewalle and Derk-Jan Dijk
}

\section{Light exposure is the major environmental factor regulating sleep and wakefulness}

Light is necessary for image formation by the visual system, but is also essential for the regulation of numerous non-imageforming functions $[1,2]$. Light is responsible for the synchronization of circadian rhythms to the external world and is able to alter the timing of these rhythms. Circadian rhythmicity is present in many processes, most notably in thermoregulation, endocrine and cardiovascular functions, sleep, alertness, and cognitive abilities. Light indirectly affects all these aspects in the long term, through its impact on circadian rhythms. Lack of light has also a negative impact on mood and repeated daily exposure to light is recommended as a therapy in seasonal affective disorders. These effects on mood, are possibly mediated through an impact on circadian rhythmicity [3]. In addition to these long-term effects, light also evokes strong and acute alterations in alertness, sleep propensity, attention, cognitive performance, cognitive brain function, body temperature, heart rate, melatonin and cortisol secretion, gene expression, and pupil constriction $[1,2,4,5]$. Since most of the long-term and acute non-image-forming impacts of light either directly or indirectly affect the quality of sleep and wakefulness, light exposure emerges as a powerful environmental factor regulating our 24-h lives (Figure 21.1).

Analyses of the effects of light on cognition showed that the effects on performance are acute and encompass executive processes, such as inhibition, shifting and updating, memory processes, including working memory and long-term memory, and attention. Thus light has a broad impact on major aspects of cognition associated with wakefulness [1,5-9] Light exposure during wakefulness has also been reported to acutely reduce electroencephalogram (EEG) delta, theta, and alpha activity as well as slow eye movements, which are all correlates of sleepiness and attention deficits $[5,9,10]$. This provides a first indication of the mechanisms involved in the effects of light on non-image-forming brain functions.

In this chapter, we will describe the physiological bases of the impact of light on non-image-forming functions before summarizing recent neuroimaging investigations that substantially increased our understanding of the brain mechanisms involved. The data show that light has a widespread impact on many brain functions and that this effect depends on the brain region and the ongoing process considered as well as on the duration and intensity of the light exposure, and time of day. The effects of light are also modulated by the health status and genotype of the participants.

\section{The non-image-forming functions of light are mediated by a photoreception system sensitive to blue light}

It has been shown in several species, including rodents, macaques, and humans, that light affects non-image-forming functions even in the absence of rods and cones, the classical visual photoreceptors [4]. This implies that another photoreceptor is involved. In addition, the ability of light to suppress melatonin secretion and subjective sleepiness, to increase body temperature, heart rate, and objective measures of alertness, and to shift circadian phase has been reported to be greatest with exposure to shorter wavelength light (at around $460-480 \mathrm{~nm}$; blue light), which is at odds with the maximal sensitivity of the classical cone-driven photopic ( $550 \mathrm{~nm}$ - green) and rod-driven scotopic ( $505 \mathrm{~nm}$ - blue-green) photoreception systems $[1,2,4,5]$.

Intrinsically photosensitive retinal ganglion cells (ipRGC) expressing the photopigment melanopsin constitute a novel class of photoreceptors only marginally involved in vision, but deeply implicated in the non-image-forming functions of light [4]. Melanopsin is necessary for the intrinsic light response with a maximal sensitivity to wavelengths around $480 \mathrm{~nm}$ (blue light). Melanopsin-expressing ipRGCs constitute the only channel through which light affects non-image-forming functions but they receive inputs from rods and cones which modulate their overall response to light. Light responses depend therefore on the state of each class of photoreceptors so that the strength and wavelength sensitivity of the impact of light on non-image-forming functions vary with irradiance level and duration of light exposure and prior light history [4].

Melanopsin-expressing ipRGCs project to the lateral geniculate nucleus and the superior colliculus, and these projections are likely to contribute to vision, but also possibly to nonimage-forming functions of light [4]. IpRGCs also project to the hypothalamus, particularly the suprachiasmatic nucleus (SCN), site of the master circadian clock, and the dorsomedial 


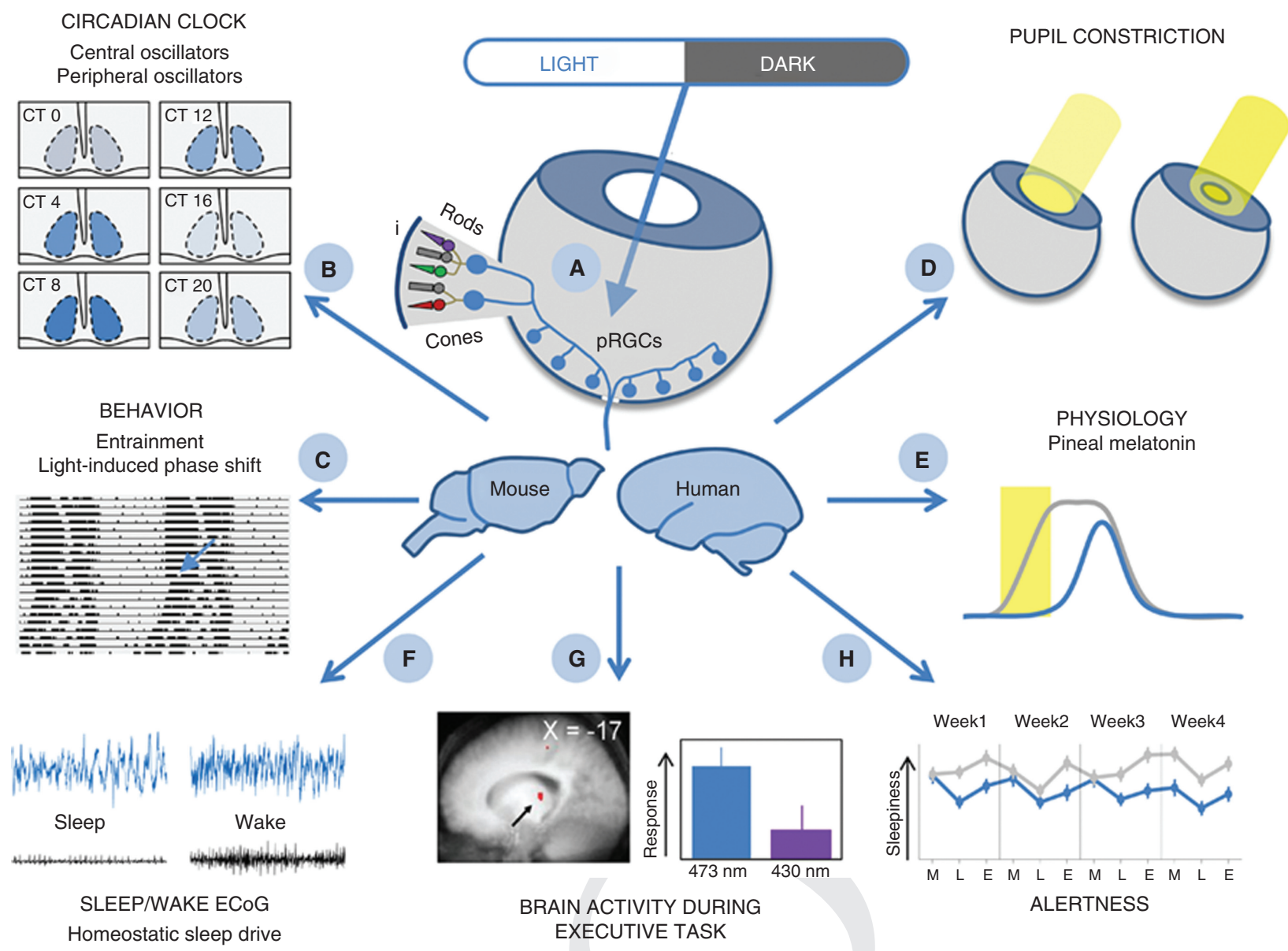

Figure 21.1 The broad non-image-forming functions of light. A diffuse network of melanopsin-expressing intrinsically photosensitive retinal ganglion cells (ipRGCs) are maximally sensitive to blue light $(\sim 48 \mathrm{~nm})$ and receive input from rods and cones (A). These cells have direct projections to the SCN, site of the circadian master clock, and regulate circadian entrainment notably through influence on gene expression (B). Light also regulates locomotor activity and can shift circadian phase (C). IpRGC connections to the pretectum mediate pupil constriction (D), and indirect input via the SCN regulates the light-induced suppression of melatonin production in the pineal (E). ipRGCs also have direct projections to sleep regulatory structures such as the VLPO and thereby modulate sleep (F). Blue light can modify cognitive brain responses $(\mathrm{G})$ and can improve alertness $(\mathrm{H})$ during the morning, lunch time, and early evening. IpRGCs are also involved in rudimentary visual functions in rodents. (Figure copied from [2].) SCN = suprachiasmatic nucleus; VLPO = ventrolaterral preoptic area; ECOG = electrocorticography.

hypothalamus (DMH), involved notably in feeding behavior and relay between the SCN and the brainstem locus coeruleus (LC) $[4,11]$. A direct projection of ipRGCs to the ventrolateral preoptic area (VLPO), and the lateral hypothalamus (LH), containing orexin neurons may mediate effects on sleep regulation [4]. Projections of ipRGCs also include the pretectum within the posterior pretectal nucleus and olivary pretectal nucleus (OPN), mediating pupillary constriction, and the lateral habenula, implicated in reward processing [4]. Finally, sparse projections to the medial amygdala, involved in emotion regulation, have been reported [12]. These widespread and numerous projections are an essential component of the brain mechanisms through which light can exert a potent and diverse impact on non-image-forming functions.

\section{Widespread impact of light on brain functions}

A recent series of neuroimaging studies in young and healthy participants investigated the impact of light on cognition, initially using positron emission tomography (PET) [13], and functional magnetic resonance imaging (fMRI) in five subsequent experiments [14-18]. The experiments were designed so that the non-image-forming role of light could be examined with as little contamination as possible from its role in image formation. All studies administered light while subjects were performing tasks in the auditory modality. In other words, care was taken not to use tasks that directly depended on the visual system. The light was administered using large uniform diffusive surfaces, thereby minimizing activation of image-forming processes. Light stimuli were long ( $40 \mathrm{~s}$ to $20 \mathrm{~min}$ ) and the effects of these stimuli were assessed during and after exposure. Comparisons were made between light of different wavelength, i.e., blue $(473 \mathrm{~nm}$ and $480 \mathrm{~nm})$, green $(527 \mathrm{~nm}$ and $550 \mathrm{~nm})$, or violet $(430 \mathrm{~nm})$, but equal photon density. This type of light exposure and assessments of its effects is very different from classical visual research in which the emphasis is on transient visual responses to small brief and contrasted stimuli.

Results demonstrated that light had a strong influence on the brain responses to an oddball paradigm task $[13,14]$, which 
mainly recruits attention, and to an n-back task [15-17], which involves continuous updating and comparing of information in working memory in addition to attention. More recently, brain responses to an emotional task, which requires the processing of negative and neutral prosody, were also reported to be affected by light exposure [18]. This latter observation extends the known acute non-image-forming role of light to the emotional domain and opens new perspectives as to how light affects mood and emotions in the long term.

These studies showed that light affected subcortical areas involved in alertness regulation. These areas included the hypothalamus, in an area encompassing the SCN and VLPO, while subjects were engaged in an oddball task [13]. Another area of the hypothalamus compatible with the $\mathrm{DMH}$ and paraventricular nucleus of the hypothalamus (PVNH), both involved in emotional responses, was affected by exposure to light specifically during the processing of negative auditory emotional stimulations (not for neutral stimuli) [18]. The dorsoposterior thalamus, in an area compatible with the pulvinar, was repeatedly shown to be affected by light exposure while performing an oddball and n-back tasks. Furthermore, an area of the brainstem compatible with the LC was affected during an n-back task [14-18]. Light also influenced responses of the hippocampus and amygdala, which are involved in long-term memory and emotion regulation.

At the cortical level, light affected areas involved in attention regulation during the exposure but also for several min during the darkness period immediately following light exposure [19]. Light-induced increases in activation were also observed in the occipital and temporal sensory cortices, likely through an increase of the top-down influence from parietal and frontal areas. Areas involved in updating and comparing of information in working memory were also affected [20]. These areas include the insula, the intraparietal sulcus (IPS) and supramarginal gyrus in the parietal cortex, and the dorso- (DLPFC) and ventrolateral prefrontal (VLPFC) cortex and frontopolar cortex (FPC) in the prefrontal cortex. According to a recent model of cognition [21], FPC and VLPFC are at the apex of the hierarchy of executive control and are involved in the more complex processes. Light appears therefore able to affect the fronto-parietal repetition loop involved in working memory including those prefrontal areas at the top of executive control. Finally, the voice area of the temporal cortex was acutely affected by light exposure specifically for the processing of negative prosody stimulations [18].

The data show that light is able to trigger widespread modulations of brain activity in subcortical and cortical areas involved in the ongoing cognitive process. The distribution of affected areas is task-dependent and can include areas at the top of executive control, or crucial for attention, but also structures at the core of emotional responses, or essential for alertness regulation.

\section{Wavelength-dependent modulation of brain responses}

Behavioral studies have shown that, similarly to other nonimage-forming functions, blue or blue-enriched light have a superior impact on cognitive performance, as compared to green light or standard white light [1,5-9]. In accordance with these behavioral studies, brain responses to auditory nback or emotional tasks were shown to be stronger with blue light exposure $(473 \mathrm{~nm}$ or $480 \mathrm{~nm}$, to which melanopsin ipRGCs and the non-image-forming system is maximally sensitive) compared to green light $(527 \mathrm{~nm}$, to which M-cones are maximally sensitive, and $550 \mathrm{~nm}$ to which the photopic visual system is maximally sensitive) and violet light $(430 \mathrm{~nm}$, to which S-cones are maximally sensitive) [15-18]. These wavelength-dependent effects were detected in subcortical areas including the hypothalamus (DMH- or PVNHcompatible area), in the brainstem (LC-compatible area), and pulvinar, but also in the IPS, DLPFC, VLPFC, FPC, as well as within the amygdala, hippocampus, insula, and temporal cortex voice-sensitive area. The superiority of blue light was observed during exposures lasting as little as $40-60 \mathrm{~s}$ and exposures lasting up to $18 \mathrm{~min}$. In the amygdala and hippocampus the superiority of blue light compared to green light $(527 \mathrm{~nm})$ can already be observed at light onset, which is an event independent of cognition [16].

These results show that the impact of light on non-imageforming function is wavelength-dependent. The superiority of blue light suggests that the classical photopic visual system is not primarily responsible for the modulation of non-imageforming brain responses and is compatible with a major contribution from melanopsin-expressing ipRGCs [4, 22]. These observations do not exclude contributions from cones, while a contribution from rods remains to be investigated. Rods have been shown to contribute to the non-image-forming function of light at a higher irradiance level than their upper functional limit for vision $[4,22]$.

\section{Duration-, intensity-, and task difficulty-dependent impact of light}

Although no dose-response studies have been carried out, greater doses of light appear to induce stronger modulations. Twenty min of bright white light triggered significant effects on brain responses, which could be detected for up to $10 \mathrm{~min}$ after light off $[13,14]$, while significant differential effects of blue and green could only be detected during an 18-min exposure, which contained at least 100 times less photons, and not during the darkness period following light exposures [15]. Moreover when reducing light duration to less than a min, brain responses to a 2-back task were mainly detected in subcortical areas including the pulvinar and a brainstem (LC-compatible) area, with little significant impact on the cortex [16]. This indicates that lightinduced modulation of brain activity can be detected in subcortical areas when light is not yet significantly affecting the cortex.

In a subsequent investigation, impacts on the cortex were more numerous and widespread when using a much more difficult 3-back task (in which the current item has to be compared to the item presented three items earlier, instead of two) [17], suggesting that the effects of light were not only dependent on light intensity and duration, in addition to its 
wavelength, but also on the difficulty of the ongoing process, with more significant cortical modulation for more demanding tasks.

Significant behavioral impacts were only found with a higher amount of light (16 to $20 \mathrm{~min}$ of bright white light) $[13,14]$. This supports the assumption that significant changes in brain responses precede significant behavioral changes and that it is only when the impact on brain function is strong enough that it is translated into behavioral changes. This assumption is supported by behavioral studies, which used similar wavelength and irradiance levels but for much longer durations, and found significant performance increase on task recruiting attention and executive functions $[1,5-9]$.

\section{The interaction between circadian and sleep homeostasis signals modulates the impact of light}

The first PET investigation of the impact of light on non-imageforming cognitive brain functions was conducted at night [13], while the subsequent fMRI studies were conducted during the daytime [14-18]. An impact of light on brain function was detected both at night and during the day. However, differences between PET and fMRI and between protocols limit the information that can be obtained from comparisons between these night and day experiments. Recent data show that time of day (morning vs. evening) and the associated changes in the interaction between circadian phase and homeostatic sleep pressure modulates the impact of light on cognitive brain function [17].

As already mentioned, the impact of light appears to be particularly pronounced under challenging conditions, which can be encountered while performing a difficult task, but also when the interaction between circadian and sleep homeostasis signals jeopardizes brain function. When comparing two fMRI sessions acquired in the morning at the same circadian phase, after a night of sleep and a night of sleep deprivation, respectively, it is in the latter condition that more widespread and more numerous light-induced modulations were observed [17]. Compared with green light $(527 \mathrm{~nm}), 1 \mathrm{~min}$ of blue light $(473 \mathrm{~nm})$ could significantly increase brain responses to a 3 back task in the FPC, DLPFC, premotor cortex, and IPS, in the morning after a night of sleep or after a night of sleep deprivation. However, following sleep loss, effects were more numerous and widespread, so that they became significant bilaterally in prefrontal and parietal cortex and also included the insula (bilaterally) and the pulvinar.

Surprisingly, no differential impact of blue and green light on brain responses could be detected with a 1-min exposure in the evening, in the so-called wake-maintenance zone (i.e., when the circadian signal maximally promotes wakefulness [23] - see Chapter 20), following a normal waking day [17]. This implies that when the endogenous drive for wakefulness is high, there is a relative decrease in the capacity of light to modulate brain activations. It appears that the interaction between circadian and sleep homeostasis, which governs our ability to maintain proper waking brain function ([23] - see Chapter 20), also has a dramatic influence on the ability of light to affect brain responses. Indeed, if homeostatic sleep pressure alone was influencing light impact, intermediate effects should be detected in the evening, as compared with effects in morning sessions after sleep and after sleep loss. In other words, light is not as stimulating in the evening, when brain responses are already maximally stimulated endogenously by the circadian system, and more light would be required to induce significant effects at this circadian phase. This assumption is supported by data showing significant impact of light on performance in the evening wake-maintenance zone using a similar irradiance level and wavelength of blue light but much longer exposures [8].

\section{The impact of light depends on genotype}

A variable number of tandem repeat (VNTR) polymorphism in the coding region of the gene PERIOD3 (PER3) predicts a difference in the buildup of homeostatic sleep pressure and individual vulnerability to the impact of sleep loss on cognitive performance [23]. One genotype $\left(P E R 3^{5 / 5}\right)$ has a faster increase of sleep pressure and is more vulnerable to sleep loss, as compared to the other $\left(P E R 3^{4 / 4}\right)$ (see Chapter 20). While the impact of light in the evening wake maintenance zone did not differ between the genotypes, blue light significantly increased brain responses in the morning following sleep loss only in PER $3^{5 / 5}$ individuals, and in the morning following a night of sleep only in $P E R 3^{4 / 4}$ individuals [17]. This constitutes the first identification, both in human and animal research, of a genetic marker of individual differences in the impact of light on non-imageforming functions.

The difference between PER3 genotypes with respect to their response to light may be related to differences in the endogenous drive for wakefulness. Because the buildup of homeostatic sleep pressure is slower in $P E R 3^{4 / 4}$ individuals they are less affected by the combination of sleep loss and an adverse circadian phase, i.e., the morning hours. $P E R 3^{4 / 4}$ individuals are therefore able to trigger endogenous compensatory brain mechanisms that maintain brain responses and recruit supplemental areas to perform a 3-back task even in the absence of light [24] (see Chapter 20). In contrast, the combination of sleep loss and an adverse circadian phase induces major reductions of activations across all parts of the cortex in $P E R 3^{5 / 5}$ individuals. Light appears therefore less able to stimulate brain responses in individuals already under great endogenous stimulation. It will provide more benefits to the genotype which is not able to maintain brain responses endogenously and is most challenged by the circadian and sleep homeostasis conditions. In addition, $P E R 3^{5 / 5}$ individuals are more likely to be morning chronotypes and to prefer performing in the morning hours [23]. The circadian amplitude in brain responses may be larger in morning types (see Chapter 20) so that in the morning following sleep they would be in optimal endogenous conditions to perform and could not benefit as much from an external light stimulation. PER3 $3^{4 / 4}$ individuals, which represent $45-50 \%$ of the general population and are more likely to be evening chronotypes, would benefit more from light in the morning after a night of sleep and significant effects could be detected. This 
hypothesis is in agreement with the previous studies, which were carried out in the morning shortly after sleep and found significant impact of light on brain responses in non-genotyped samples [14-16].

\section{Light modifies the functional organization of the brain}

Simple psychophysiological interaction analyses provided further insight on how light is able to modulate ongoing brain activity. While performing a 3-back task recruiting attention, working memory, and executive function, blue light increases the functional connectivity between the pulvinar and DLPFC, premotor cortex, and IPS, as well between the DLPFC and IPS (unpublished analyses of [17]). During an emotional auditory task using voice stimulation, the light-activated network is different and comprises the hypothalamus (DMH- or PVNHcompatible location), amygdala, and the voice-sensitive area of the temporal cortex [18]. The latter result was specific for the processing of emotional stimuli and was not observed for neutral stimuli. These data show that (blue) light modifies information flow between areas essential for a given ongoing task and suggest that the interactions between brain areas are optimized.

\section{Summary and scenario of the brain mechanisms}

In accordance with animal research, the neuroimaging results presented here are compatible with a scenario in which light would first influence subcortical structures involved in arousal regulation before significantly affecting the cortical areas involved in the ongoing non-image-forming process (Figure 21.2). These subcortical structures include the hypothalamus, within the SCN, but also possibly the VLPO, DMH, and PVNH. The SCN and DMH could in turn transfer light information to numerous brain sites through their numerous projections, which include the brainstem LC, to which they are functionally connected [11]. The LC constitutes the major source of norepinephrine of the organism and has a broad impact on alertness and cognition through its numerous projections to the thalamus and most of the cortex. However, other (aminergic) nuclei of the ascending arousal system could possibly be recruited as well. A pulvinar-compatible area of the thalamus appears also to be recruited at light onset but also throughout the $60 \mathrm{~s}$ to $20 \mathrm{~min}$ of exposure. The pulvinar, which has been suggested to mediate the impact of alertness on cognition $([24,25]$ - see Chapter 20), is a major relay between cortical areas and an impact of light in that area could facilitate information flow within the cortex, as suggested by functional connectivity changes identified for a task involving attention, working memory, and executive functions. If sufficient, this subcortical impact would then significantly affect the cortical area recruited for the ongoing task, which could in turn affect behavior. Behavioral measures would only be significantly affected after prolonged light exposure either because the cortical impact requires time for the effect to be transferred to behavior or because behavioral measures are less sensitive than neuroimaging techniques, or probably both.

Several factors modulate the effects described in this scenario including light intensity, duration, and wavelength, and possibly task difficulty. The interaction between circadian and

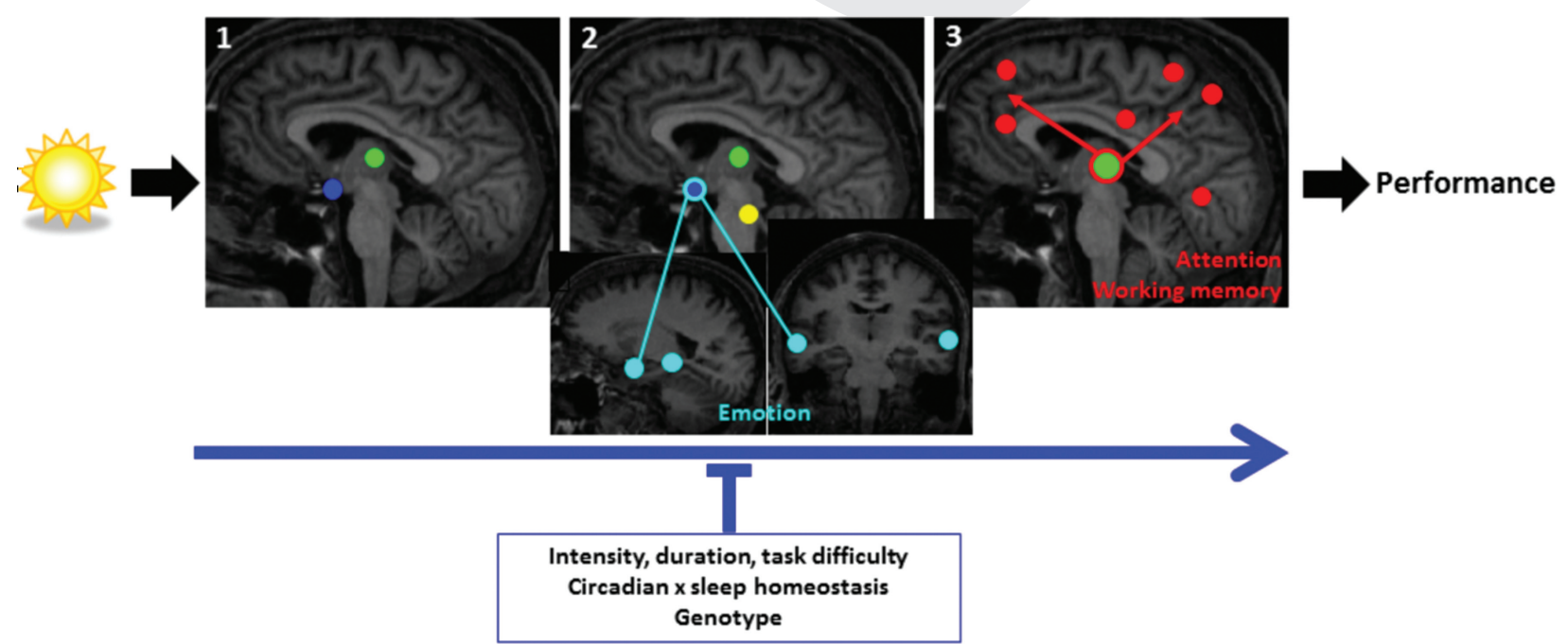

Figure 21.2 Schematic representation of the brain mechanisms involved in the non-image-forming impact of light on cognitive brain responses. (1) Responses at light onset are found within the hypothalamus (blue) and pulvinar (green) (and amygdala and hippocampus, not shown); (2) within the first seconds of the exposure, responses are found mainly in subcortical and cortical structures involved in alertness regulation (hypothalamus, brainstem (green), pulvinar); (3) late responses are detected at the cortical level in areas involved in the ongoing cognitive process and can subsequently affect performance. For attention/working memory/executive tasks (yellow) a network of areas around the pulvinar and including prefrontal and parietal areas appear to mediate the impact of light on alertness and cognition. For emotional responses to vocal stimuli (light blue), the network involves the hypothalamus, amygdala, and voice-sensitive area of the temporal cortex. Light seems to have a swifter impact on emotional cortical responses than attentional/working memory/executive responses. The impact of light is stronger with higher intensity, longer duration, and shorter wavelength (blue) light exposures, and seems also more pronounced when the participant performs a more difficult cognitive task. Time of day and the associated changes in the interaction between circadian and sleep homeostasis signals and PERIOD3 genotype modulate the impact of light. (Adapted with permission from [5].) 


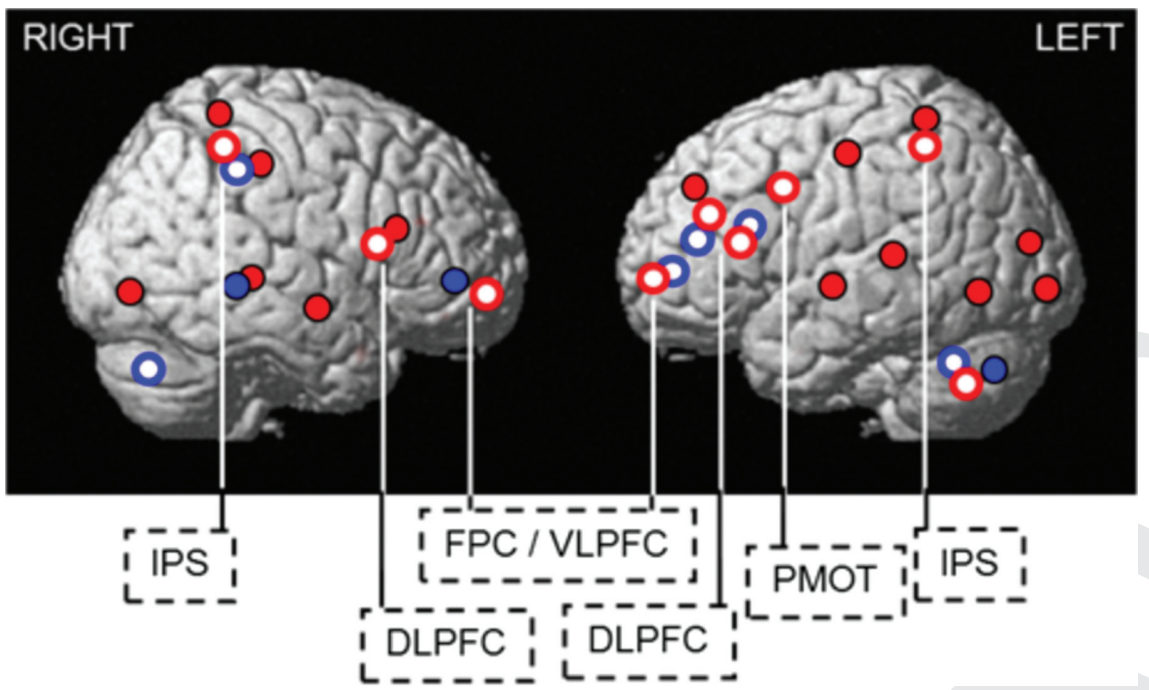

Figure 21.3 Blue light increases cognitive brain responses in locations showing in darkness decreased activation in $P E R 3^{5 / 5}$ or compensatory recruitment in PER $3^{4 / 4}$ following sleep loss. Compensatory increase in activation in the morning hours after 25 hours of wakefulness in PER3 $3^{4 / 4}$, found notably in the ventrolateral prefrontal cortex, temporal cortex, cerebellum, and thalamus (thalamus not shown). Decreases in activation in the morning hours after 25 hours of wakefulness in PER $3^{5 / 5}$, observed notably in the occipital, temporal, parietal, and lateral prefrontal cortices. Blue light-induced increase in activations after 25 hours of wakefulness in $P E R 3^{5 / 5}$ (thalamus not shown). Blue light-induced increase in activity after 1.5 hours of wakefulness in PER $3^{4 / 4}$.

$\mathrm{DLPFC}=$ ventrolateral/dorsolateral prefrontal cortex; FPC/ $\mathrm{VLPFC}=$ frontopolar/ventrolateral prefrontal cortex; IPS = intraparietal sulcus; $\mathrm{PMOT}=$ premotor cortex (Copied with permission from [17].)

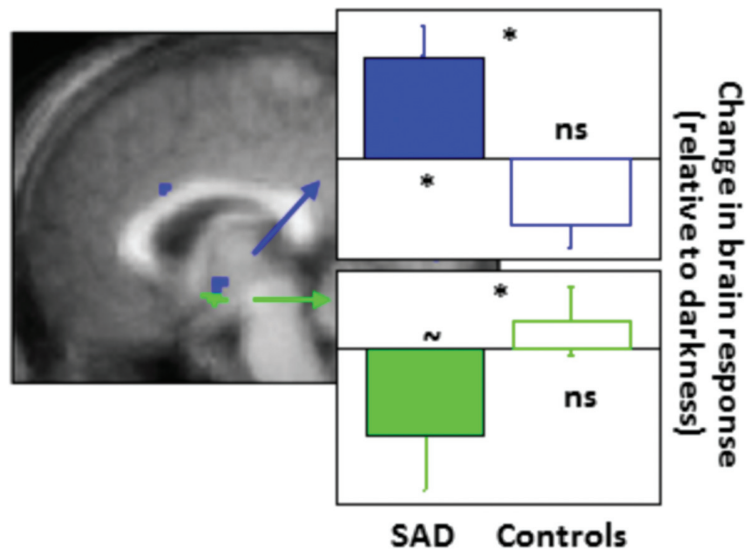

Figure 21.4 Hypothalamic responses to light as a neurobiological substrate of SAD. The impact of blue and green light exposure on the brain responses to auditory emotional stimuli is significantly different in the hypothalamus ( $\mathrm{LH}$ - or PVNH-compatible area) of patients with SAD compared with healthy controls. Graphs: change in brain response under blue $(480 \mathrm{~nm})$ and green $(550 \mathrm{~nm})$ light exposures. (Modified with permission from [26].) $* p<0.05$ after correction for multiple comparisons within a group or between group; $\sim p=0.07$ after correction for multiple comparisons; ns = net significent.

sleep homeostasis signals and PER3 genotype can amplify or diminish the non-image-forming impact of light on cognitive brain function. The impact of light is also task specific and is primarily detected in areas involved in the ongoing process. The type of cognitive function is also a crucial determinant of the effect of light. For attention, working memory, and executive functions light effects appear to be mediated through a network in which the pulvinar plays a central role but which also includes prefrontal and parietal higher associative areas. For tasks triggering emotional responses, effects within cortical areas specially devoted to decoding the emotional content of a particular type of stimulus (e.g., the voice-sensitive area of the temporal cortex for voice stimulation) appear to be quick and mediated through a network based on hypothalamus and amygdala interactions.
Importantly, the impact of light appears to be related to the activation pattern in the absence of light. For an auditory 3-back task, an impact of light is found in areas that undergo decreased activations or compensatory recruitment during sleep loss in darkness, particularly higher associative areas (Figure 21.3) [17, 24]. It seems therefore that there is an overlap between the endogenous brain mechanisms regulating wakefulness during a normal waking day and following sleep loss, and the mechanisms triggered by an external activating agent such as exposure to light.

\section{Hypothalamic response to light as a neurobiological marker of psychiatric status}

Seasonal affective disorder (SAD) is a major depressive disorder with a recurrent seasonal occurrence and most probably related to the lack of light exposure in fall/winter [3]. It has been suggested that the ability of light therapy to improve mood in $\mathrm{SAD}$ relates to abnormal circadian regulation or abnormal retinal physiology in patients [3]. The therapeutic effects of light may also be mediated through its acute ability to recruit a specific network of brain areas involved in emotional responses. This could in turn result in a better emotional regulation and improved mood.

Data acquired in patients suffering from SAD show that a posterior area of the hypothalamus, presumably including the $\mathrm{LH}$ containing orexin neurons, or the $\mathrm{PVNH}$, present increased activations to emotional vocal stimulation under blue light $(480 \mathrm{~nm})$ illumination, but decreased activation under exposure to green light $(550 \mathrm{~nm})$ (Figure 21.4) [26]. These effects were not observed in healthy controls (significant difference between patients and controls). Since the hypothalamus, and particularly the $\mathrm{LH}$, contributes to emotion, sleep/wake, and metabolism regulation, which are all disrupted to some extent in SAD, these light-induced activations may be of some interest. However, further research is required to elucidate whether this abnormal hypothalamic response to light constitutes a neurobiological indicator of how lack of light triggers SAD or 
how light therapy leads to remission, or if it is a secondary marker of a more central phenomenon.

\section{Conclusions and perspectives}

The data reviewed here indicate that exposure to light has a powerful impact on brain activity recruited by diverse aspects of cognition, so that the quality of brain functions associated with wakefulness are modulated. Several lines of evidence now suggest that exposure to light also affects subsequent sleep such that increased blue light exposure during the day can improve nocturnal sleep quality [27] and too much light in the evening disrupts sleep [28]. These effects are likely to be mediated by melanopsin, and sleep and the circadian system are so sensitive to light that ordinary room light exposure in the evening will impact circadian physiology and subsequent sleep [6, 27, 29]. This appears particularly relevant since melanopsin ipRGCs have been shown not only to acutely affect sleep and wakefulness, but also to have an impact on sleep homeostasis: melanopsin depletion in rodents reduced slow wave sleep rebound after sleep deprivation [30].

Understanding how light can be used to improve wakefulness and be modified to positively affect sleep could be of great interest for several segments of the general population: shift workers and jet-lagged people, sleeping and waking at the wrong circadian time; older and middle-aged people, with earlier wake times, decreased sleep quality [31], and less able to shift their circadian phase in response to light [32]; Alzheimer patients, who benefit from light and melatonin administration [33]; or psychiatric patients for whom light therapy ameliorates symptoms, as demonstrated for an increasing number of disorders [3,34]. Currently, requirements for indoor lighting are primarily focused on visual acuity but should also consider the non-image-forming functions of light so that we are not "blue-light deprived," particularly in winter, when our exposure to blue light decreases [35].

\section{Acknowledgements}

We thank the members of the Cyclotron Research Centre of the University of Liège and of the Functional Neuroimaging Unit and Center for Advanced Research in Sleep Medicine of the University of Montreal for their help in realizing the studies reviewed here. These studies were supported by the Belgian Fonds National de la Recherche Scientifique (FNRS), Fondation Médicale Reine Elisabeth (FMRE), University of Liège, Interuniversity Attraction Pole (PAI/IAP) P6/29, by the Wellcome Trust-GR069714MA, and Canadian Institute for Health Research. GV is supported by the FNRS. DJD has received research support from Philips Lighting (the Netherlands) but this research is distinct from the neuroimaging data reviewed in this manuscript. The authors declare no other competing conflicts of interest.

\section{References}

1. Chellappa SL, Gordijn MC, Cajochen C. Can light make us bright? Effects of light on cognition and sleep. Prog Brain Res. 2011;190:119-33.

2. Dijk DJ, Archer SN. Light, sleep, and circadian rhythms: together again. PLoS Biol. 2009; 7:e1000145.

3. Westrin A, Lam RW. Seasonal affective disorder: a clinical update. Ann Clin Psychiatry. 2007;19:239-46.

4. Schmidt TM, Chen SK, Hattar S. Intrinsically photosensitive retinal ganglion cells: many subtypes, diverse functions. Trends Neurosci. 2011;34:572-80.

5. Vandewalle G, Maquet P, Dijk DJ. Light as a modulator of cognitive brain function. Trends Cogn Sci. 2009;13:429-38.

6. Cajochen C, Frey S, Anders D, et al. Evening exposure to a light-emitting diodes (LED)-backlit computer screen affects circadian physiology and cognitive performance. J Appl Physiol. 2011;110:1432-8.

7. Chellappa SL, Steiner R, Blattner P, et al. Non-visual effects of light on melatonin, alertness and cognitive performance: can blue-enriched light keep us alert? PLoS One. 2011;6:e16429.

8. Cajochen C, Munch M, Kobialka S, et al. High sensitivity of human melatonin, alertness, thermoregulation, and heart rate to short wavelength light. J Clin Endocrinol Metab. 2005;90:1311-16.

9. Lockley SW, Evans EE, Scheer FAJL, et al. Short-wavelength sensitivity for the direct effects of light on alertness, vigilance, and the waking electroencephalogram in humans. Sleep. 2006;29:161-8.

10. Cajochen C, Zeitzer JM, Czeisler CA, et al. Dose-response relationship for light intensity and ocular and electroencephalographic correlates of human alertness. Behav Brain Res. 2000;115:75-83.

11. Aston-Jones G, Cohen JD. An integrative theory of locus coeruleusnorepinephrine function: adaptive gain and optimal performance. Annu Rev Neurosci. 2005;28:403-50.

12. Hattar S, Kumar M, Park A, et al. Central projections of melanopsin-expressing retinal ganglion cells in the mouse. J Comp Neurol. 2006;497:326-49.

13. Perrin F, Peigneux P, Fuchs S, et al. Nonvisual responses to light exposure in the human brain during the circadian night. Curr Biol. 2004;14:1842-6.

14. Vandewalle G, Balteau E, Phillips C, et al. Daytime light exposure dynamically enhances brain responses. Curr Biol. 2006;16:1616-21.

15. Vandewalle G, Gais S, Schabus M, et al. Wavelength-dependent modulation of brain responses to a working memory task by daytime lightexposure. Cereb Cortex. 2007;17:2788-95.

16. Vandewalle G, Schmidt C, Albouy G, et al. Brain responses to violet, blue, and green monochromatic light exposures in humans: prominent role of blue light and the brainstem. PLoS One. 2007;2:e1247.

17. Vandewalle G, Archer SN, Wuillaume C, et al. Effects of light on cognitive brain responses depend on circadian phase and sleep homeostasis. J Biol Rhythms. 2011;26:249-59. 
18. Vandewalle G, Schwartz S, Grandjean D, et al. Spectral quality of light modulates emotional brain responses in humans. Proc Natl Acad Sci U S A. 2010;107:19549-54.

19. Corbetta M, Shulman GL. Control of goal-directed and stimulus-driven attention in the brain. Nat. Rev. Neurosci.. 2002;3:201-15.

20. Collette F, Hogge M, Salmon E, et al. Exploration of the neural substrates of executive functioning by functional neuroimaging. Neuroscience. 2006;139:209-21.

21. Koechlin E, Hyafil A. Anterior prefrontal function and the limits of human decision-making. Science. 2007;318:594-8

22. Lall GS, Revell VL, Momiji H, et al. Distinct contributions of rod, cone, and melanopsin photoreceptors to encoding irradiance. Neuron. 2010;66:417-28.

23. Dijk DJ, Archer SN. PERIOD3, circadian phenotypes, and sleep homeostasis. Sleep Med Rev. 2010;14:151-60.

24. Vandewalle G, Archer SN, Wuillaume C, et al. Functional magnetic resonance imaging-assessed brain responses during an executive task depend on interaction of sleep homeostasis, circadian phase, and PER3 genotype. J Neurosci. 2009;29:7948-56.

25. Portas CM, Rees G, Howseman AM, et al. A specific role for the thalamus in mediating the interaction of attention and arousal in humans. J Neurosci. 1998;18:8979-89.

26. Vandewalle G, Hebert M, Beaulieu C, et al. Abnormal hypothalamic response to light in seasonal affective disorder. Biol Psychiatry. 2011;70:954-61.

27. Viola AU, James LM, Schlangen LJ, et al. Blue-enriched white light in the workplace improves self-reported alertness, performance and sleep quality. Scand J Work Environ Health. 2008;34:297-306.

28. Santhi N, Thorne HC, van der Veen DR, et al. The spectral composition of evening light and individual differences in the suppression of melatonin and delay of sleep in humans. J Pineal Res. 2011;53:47-59.doi: 10.1111/j.1600079X.2011.00970.x.

29. Gooley JJ, Chamberlain K, Smith KA, et al. Exposure to room light before bedtime suppresses melatonin onset and shortens melatonin duration in humans. J Clin Endocrinol Metab. 2011;96:E463-72.
30. Tsai JW, Hannibal J, Hagiwara G, et al. Melanopsin as a sleep modulator: circadian gating of the direct effects of light on sleep and altered sleep homeostasis in Opn4(-/-) mice. PLoS Biol. 2009;7:e1000125.

31. Gaudreau H, Morettini J, Lavoie HB, et al. Effects of a 25-h sleep deprivation on daytime sleep in the middle-aged. Neurobiol Aging. 2001;22:461-8.

32. Duffy JF, Zeitzer JM, Czeisler CA. Decreased sensitivity to phase-delaying effects of moderate intensity light in older subjects. Neurobiol Aging. 2007;28:799-807.

33. Riemersma-van der Lek RF, Swaab DF, Twisk J, et al. Effect of bright light and melatonin on cognitive and noncognitive function in elderly residents of group care facilities: a randomized controlled trial. JAMA. 2008;299:2642-55.

34. Terman M, Terman JS. Light therapy for seasonal and nonseasonal depression: efficacy, protocol, safety, and side effects. CNS Spectr. 2005;10:647-63.

35. Thorne HC, Jones KH, Peters SP, et al. Daily and seasonal variation in the spectral composition of light exposure in humans. Chronobiol Int. 2009;26:854-66. 